\title{
Detección de nudos críticos para la disminución del tiempo de espera en atención de prolapso de órganos pélvicos en hospital público chileno
}

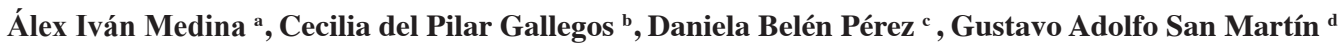 \\ ${ }^{\text {a }}$ Doctor en Finanzas y Contabilidad. Magíster en Administración y Dirección de Empresas. \\ Facultad de Ciencias Empresariales, Universidad del Bío-Bío \\ alex@ubiobio.cl iD https://orcid.org/0000-0002-4072-1975

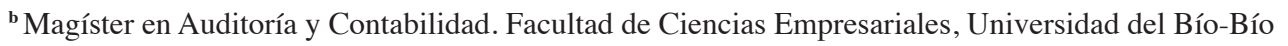 \\ ${ }^{\mathrm{c}}$ Contadora pública y auditora. ${ }^{\mathrm{d}}$ Contador público y auditor.
}

DOI 10.22517/25395203.15181

\section{Resumen}

Introducción: El problema que se presenta en la atención de salud en los hospitales públicos es la alta cantidad de pacientes, generando un sostenido crecimiento en las listas de espera y provocando un aumento del tiempo de espera, generando un impacto negativo en los usuarios.

Objetivo: Mejorar el proceso clínico de atención del prolapso de órganos pélvicos de un hospital público chileno.

Metodología: Se identifican nudos críticos mediante diagramas de flujo, realizando un diagnóstico del proceso desde el ingreso del paciente hasta su alta del hospital.

Resultados: Se identifican cada uno de los puntos críticos y se proponen soluciones para agilizar el proceso y con ello mejorar la atención a las usuarias, lo que en definitiva llevó a una disminución del tiempo de espera y, lo más relevante, a una disminución en la cantidad de personas en la lista de espera.

Palabras clave: Evaluación de procesos, atención hospitalaria, prolapso.
Detecting critical knots to decrease the waiting time when caring the pelvic organ prolapse in a chilean public hospital

\begin{abstract}
Introduction: The high number of patients is a common issue in public hospitals that causes a negative impact on the users due to the increment in the waiting lists and in the waiting time to be treated.
\end{abstract}

Objective: To improve the clinical process for caring pelvic organ prolapse in a chilean public hospital.

Methodology: The methodology was focused on identifying critical knots using flowcharts and diagnosing the patient's process from their admission until their discharge from the hospital.

Results: Some solutions were proposed for each critical point identified so as to accelerate the process, thus improving users' health caring. As a result, the high number of people in the waiting lists and the waiting time decreased substantially.

Key words: Evaluation process, hospital care, prolapse. 


\section{Gestión de proceso de listas clínicas de espera}

Uno de los objetivos estratégicos establecidos en la Estrategia Nacional de Salud 2011-2020 es el acceso y calidad de la atención de salud, particularmente en la reducción de listas de espera y la disminución de la insatisfacción usuaria. En el caso de las listas de espera, la meta es "aumentar a 90\% la proporción de Servicios de Salud con adecuada razón de resolución de listas de espera excesivamente prolongadas" ${ }_{(1)}$, lo que lleva a la mejora en los procesos clínicos y administrativos como una forma de aportar al cumplimiento de dichos objetivos sanitarios.

Si bien a nivel internacional existen diversos estudios descriptivos de las listas de espera que abordan diferentes variables, principalmente enfocados en tiempos de espera y número de pacientes en espera, en Chile estos estudios son claramente escasos.

Se han planteado diversos modelos de intervención para limitar los efectos de las listas de espera, tales como aumentar la productividad en el sector público, reformando el tipo contractual de los especialistas, utilizando recursos privados, entre otros, en donde la mayoría de ellos han apuntado al aumento de recurso para el financiamiento. Sin embargo, la gran limitación de los sistemas de información sobre listas radica en la visión compartimentada de las mismas; no se ven procesos, sino sucesos independientes en cada parte del proceso. Así, un paciente que es visto en atención primaria debe esperar una cantidad considerable de tiempo hasta llegar a la consulta externa en la primera visita; de allí en adelante la espera aumenta entre procesos tales como la cita con el especialista, la inclusión en la lista de espera para la intervención y el postoperatorio ${ }_{(2)}$.

Por su parte, los tiempos de espera para intervenciones quirúrgicas se pueden interpretar, erróneamente, como un simple desajuste entre la demanda y la oferta de un determinado procedimiento ${ }_{(3)}$. Si así fuera, sólo se debería ir aumentando los recursos hasta terminar con las listas, pero el problema es más profundo que eso. También están de acuerdo en este planteamiento las unidades de investigación del Hospital de Basurto y del Hospital de Galdakao, ambos de España, señalando que existen muchas evidencias de que el aumento aislado de los recursos no es la solución, ya que el tiempo medio de espera disminuye, pero la lista se alarga por el tiempo de espera. Serán necesarias, pues, otras medidas de gestión.
Ahora bien, hay que tener en cuenta que para el abordaje de los tiempos de espera no hay fórmulas únicas, pero una de ellas está en la mejora en la organización de los recursos de soporte del hospital para atender las diversas patologías, más allá del simple incremento de los recursos ${ }_{(4)}$, lo cual se espera pueda lograr una propuesta de reducción del tiempo de espera, con mejoras en la resolutividad y mayor satisfacción de los usuarios y las usuarias.

Para ello, es necesario incorporar herramientas orientadas a la gestión por procesos hospitalarios como una alternativa de solución para contribuir al aumento del rendimiento hospitalario, de manera que facilite la detección de procesos defectuosos o procesos que no contribuyan al logro de los objetivos. Es importante reconocer que existen aspectos relevantes a la hora de realizar una buena gestión por procesos, en primer lugar, contar con buenos equipos de gestión, capacitados para identificar y solucionar problemas, segundo, el hospital debe ser capaz de manejar las exigencias que demandan determinados procesos y, por último, tener en cuenta los recursos con los que se cuenta.

La gestión por procesos se basa en un sistema integrado de procesos que prioriza la calidad y la seguridad en la atención, optimizando la comunicación, el cuidado y la promoción del equipo de salud, el apoyo en guías de práctica clínica, la historia clínica única y la evaluación permanente de los resultados, con el objetivo de mejora continua ${ }_{(5)}$. Para que pueda tener buenos resultados, es necesario que la gestión de procesos sea auto gestionada, entendiéndose por autogestión "El sistema de organización de una empresa según el cual los trabajadores participan en todas las decisiones”, según Seguel y Paravic ${ }_{(6)}$ en donde "la información debe fluir vertical y horizontalmente" de manera que todos estén involucrados con la organización y el logro de los objetivos.

Hay que tener en cuenta que los procesos de gestión no siempre son el eje central de las demoras en los tiempos de espera de los pacientes para recibir atención o un determinado tratamiento, sino que también influye la cantidad de personas que estén ingresadas en el sistema hospitalario o bien por un determinado procedimiento mal ejecutado. Cabe señalar que aunque el aumento de los recursos es importante, no es en sí la solución, pero si garantizan la equidad entre todos los ciudadanos en el acceso a los servicios de $\operatorname{salud}_{(7)}$.

\section{Prolapso y lista de espera}

En lo que respecta a las disfunciones femeninas del piso pélvico (DFPP), éstas encierran un grupo de condiciones patológicas de la mujer, incluyen la incontinencia urinaria y el prolapso de órgano pélvico, así como alteraciones sensitivas del tracto urinario inferior, síndromes de dolor relacionados con 
órganos pélvicos, entre otras ${ }_{(8)}$. Según estudios de prevalencia, aproximadamente $23,7 \%$ de las mujeres presenta uno o más de estos desórdenes, cuyo impacto en la salud femenina es importante y representa uno de los motivos de consulta más frecuentes en el área de la urología y ginecología ${ }_{(9)}$.

Con respecto al tipo de consultas ginecológicas recibidas en el Hospital de San Carlos (HSC), la más común y la que posee más listas de espera es la de prolapso de órganos pélvicos (POP), entendiéndose por tal al "descenso de una o más de las paredes vaginales, cuello del útero, o cúpula vaginal”, y la "incontinencia de orina como la observación de pérdida involuntaria de orina durante el examen físico" ${ }_{(10)}$ y cuya prevalencia general en la población puede ascender hasta el $41,1 \%$ y se encuentra asociada a factores tales como la edad, el número de partos y el índice de masa corporal ${ }_{(8)}$.

La elección de esta patología en particular, se hizo considerando su incidencia en las listas de espera que no están consideradas en las Garantías Explícitas de Salud (GES) en Chile y atendidas en el HSC, con una ocurrencia de $17 \%$ del total de listas de espera no GES ${ }_{(11)}$ con un tiempo de espera de solución de 564 días, en promedio, en los casos resueltos y de 924 días, en promedio, en los casos no resueltos hasta el $31.05 .15_{(11,12)}$, lo cual es un tiempo de espera muy prolongado para recibir la atención de salud requerida, frente al problema de salud que les aqueja, lo que provoca una fuerte insatisfacción en las usuarias y además incide significativamente en el aumento de las mujeres que están en las listas de espera.

Desde un punto vista clínico, el prolapso genital es la protrusión de los órganos genitales o parte de ellos a través del introito vaginal. Puede o no ir acompañado de incontinencia urinaria y fecal. El prolapso genital constituye la causa más frecuente de cirugía ginecológica en mujeres de más de 50 años de edad. El diagnóstico se realiza mediante exámenes complementarios como la anamnesis y examen clínico, la ecotomografía ginecológica (eventual estudio ultrasonográfico del piso pelviano) y examen de orina completo y urocultivo (eventual estudio urodinámico), todo lo cual implica diversas actividades como parte del proceso de atención y demoras en el diagnóstico.

El manejo terapéutico pasa por un tratamiento esencialmente quirúrgico. Las formas leves o asintomáticas solo se controlan, con eventual tratamiento kinésico. Dados los diferentes tipos de prolapso, también requieren diferentes tipos de cirugía. Cabe mencionar que la cirugía del piso pélvico es una de las más frecuentes en ginecología ${ }_{(12)}$. La incidencia de cirugía por prolapso de órganos pélvicos (POP) aumenta con la edad de la mujer, por lo que se espera que la prevalencia vaya en aumento. Dado que el POP causa un gran impacto en la calidad de vida de la mujer, es motivo frecuente de consulta, que además genera presión en la solución quirúrgica y demora en su solución.

Por tanto, el objetivo de este artículo es exponer como se logró contribuir a mejorar los procesos que inciden en la disminución del tiempo de espera en la atención de la patología prolapso de órganos pélvicos o genital del hospital de San Carlos, a través de la identificación de nudos críticos en sus procesos.

\section{Aportes de la reingeniería de procesos a la gestión clínica}

En lo que respecta a la reingeniería de procesos, se encuentra a Osorio y Paredes ${ }_{(13)}$, quienes buscaron la forma de realizar una reingeniería de procesos en un hospital público, con el fin de entender cada proceso y desarrollar estrategias de mejoras, señalando que ante cualquier propuesta de mejora hay que tener en cuenta 3 puntos de vista para su ejecución: 1) factibilidad operativa; 2) factibilidad técnica y 3) factibilidad económica. Como resultado del estudio, se obtuvo que gran parte del personal de enfermería dedicaban hasta el $60 \%$ de su tiempo en rellenar volantes, planillas de turnos, etiquetas de identificación de pacientes, etc., es decir, trabajo distinto al que constituye su principal actividad profesional. Por otra parte, el desaprovechamiento de tiempos productivos, junto con las altas listas de espera, conducían a una escasa disposición de tiempo y medios para la formación permanente, lo que se traducía en unas bajas expectativas de progreso profesional, asociadas a una sensación de cierta frustración y desmotivación personal. Además, se establece que no es necesario el contacto telefónico entre Admisión y la planta de hospitalización para conocer la disponibilidad de camas si los historiales clínicos estuvieran al alcance mediante una red en cualquier momento y así disminuir los tiempos y los desplazamientos innecesarios.

Bernardes et al. ${ }_{(14)}$ comentan que un hospital en Brasil estuvo cerca de 30 años bajo un modelo administrativo centrado en la administración clásica. Después, pasó a ser trabajada la propuesta de descentralización de la estructura administrativa, mediante la adopción de los principios de la gestión compartida. El hospital fue entonces segmentado en trece unidades funcionales. Los autores concluyen que en el modelo descentralizado, los problemas tienden a ser solucionados con mayor agilidad y adecuación, principalmente porque los profesionales tienen mayor autonomía y poder para que la resolutividad sea alcanzada de forma rápida. También destacan que en la medida en que las personas participan de las propuestas de solución, hay tendencia para alcanzar una mayor adecuación en la resolución, ya que los individuos experimentan esos problemas directamente en su trabajo cotidiano.

En lo que respecta a las listas de espera, Martí (7) plantea que es necesario gestionar las listas, tanto en el plano social, de 
administración sanitaria y, sobre todo, en el ámbito del centro sanitario. Menciona que la gestión clínica en los servicios y la gestión de cada profesional, deben ser priorizados por gravedad, probabilidad de mejora y criterios sociales. Destaca que la Administración sanitaria debe ser capaz de fijar tiempos máximos de espera razonables para cada tipo de procedimiento que esté en su cartera de servicios, garantizando al usuario el cumplimiento de esta espera, por lo cual deberá adecuar sus recursos a este compromiso. Además plantea que para entrar a pacientes en lista de espera y ordenarlos en ella, serán necesarios criterios de efectividad de los procedimientos, es decir, de indicación y valoración de mayor posibilidad de beneficio (costo-efectividad); criterios de mayor necesidad, por gravedad de la patología, pero también por asociación de criterios sociales, y finalmente criterios de orden de llegada.

La problemática va más allá del tiempo de espera, ya que hay un momento en el cual la espera por la resolución de un problema de salud se transforma en excesiva y potencialmente dañina para la salud y el bienestar del paciente. Una espera excesiva puede resultar de un impacto importante tanto en la salud como en términos económicos y una baja considerable en la calidad de vida del paciente durante la espera. Sumado a lo anterior, los profesionales de la salud también se sienten afectados frustrándose por no dar solución a los problemas para los cuales fueron preparados.

Como lo mencionan Del Castillo y De Portugal ${ }_{(15)}$, el médico internista en el entorno hospitalario es decisivo para favorecer una atención sanitaria centrada en las necesidades globales de la persona y es capaz de mantenerse como referente ante enfermedades específicas o nuevas. Sin embargo, internistas, cirujanos y anestesistas son los médicos con mayor déficit en red pública ${ }_{(16)}$. Según Mañalich ${ }_{(17)}$, "el debate hasta ahora se ha centrado en la falta de incentivos ¿Cuáles? Mejorar las remuneraciones en el sistema público; disponer de hospitales modernos con equipamiento donde puedan desarrollar la práctica clínica; ampliar los programas de especialización y de perfeccionamiento y realizar una adecuada planificación junto con el Ministerio de Salud de los planes de formación, tomando en cuenta los cambios demográficos de la población. Sin embargo, el solo aumento de las rentas en el sector público no garantiza que haya cambios en la distribución de los médicos privado-públicos"

Del Castillo y Khosravi ${ }_{(18)}$, mencionan que la administración clínica y la gestión deben buscar un punto de encuentro para compartir conocimientos y proporcionar la mejor medicina posible con los recursos disponibles. Logrando de la mejor forma proporcionar al médico información completa de la máxima calidad, a fin de optimizar los recursos y los tiempos.

Las entidades públicas por lo general, constan de innumerables filas o colas de espera, esto ocurre por el bajo costo para el usuario y en la mayoría de los casos la gratuidad, por tanto, la cola ya es parte del sistema de salud público.

Dentro de los factores que pueden intervenir en el tiempo de espera del usuario en el sistema de salud público están: 1) aumento de la demanda en los últimos años; 2)escasez de oferta; 3) déficit de planificación; 4) gestión y forma de pago ${ }_{(19)}$. Marti establece como posibles soluciones, necesidad de cambios en la planificación y la gestión de los servicios sanitarios, necesidad de planes de gestión globales de las listas de espera y también la necesidad de mejora de la gestión clínica $_{(19)}$. Sin dejar de lado la capacidad que tenga el sistema: tanto en recursos humanos como en recursos físicos, camas, pabellones, equipamientos, etc.

A todo lo anterior se suma el hecho de que es cada vez más frecuente la consulta ginecológica por problemas resultantes del prolapso genital y mayor las expectativas y exigencias en relación a su tratamiento, el aumento sostenido en la esperanza de vida implica un mayor número de mujeres susceptibles de desarrollar prolapso ${ }_{(20)}$.

\section{Metodología.}

Investigación descriptiva mediante estudio de caso, usando como fuentes de información primaria entrevistas y observación directa, con ello se diagnosticó de manera presencial los procesos clínicos y administrativos que se encuentran involucrados en la especialidad de Ginecología del Hospital de San Carlos, en la patología prolapso de órganos pélvicos (POP). Las fuentes secundarias fueron libros, bases de datos, datos estadísticos, informes, páginas web, etc.

Con la información recopilada se construyeron diagramas de flujo con el programa Bizagi Modeler y a través de focos grupales compuestos por personal del HSC y los investigadores, se detectan los nudos críticos presentes en los procesos y las posibles soluciones a ellos.

Cabe mencionar que se tomaron los resguardos éticos necesarios para proteger la confidencialidad de la información, tanto del Hospital como de los pacientes de Prolapso de Órganos Pélvicos.

\section{Resultados.}

\subsection{Diagnóstico}

En el HSC, para acceder a la atención de un médico especialista (ver flujo $\mathrm{N}^{\circ} 1$ ), en este caso un Ginecólogo, existen 3 instancias diferentes: la primera de ellas es la Atención Primaria de Salud (APS) que consiste en la generación de una 
interconsulta médica del consultorio o centro de atención de primera instancia al cual pertenece el usuario. La segunda forma de ingreso a la interconsulta es a través del Consultorio Adosado de Especialidades (CAE), aquí ingresa la persona por una dolencia no identificada y es el especialista tratante quien debe derivar al paciente a un ginecólogo, la tercera forma para generar una citación es mediante Asistencia Pública (AP) de urgencia en donde ingresa por una urgencia médica, y posteriormente, la matrona entrega interconsulta de especialidad.

Adicional a lo anterior, para aquellas personas que provienen de consultorios que no están incluidos en los sistemas actuales, uno de los factores de demora en el proceso, es que son ingresados en un sistema distinto del resto de los pacientes, esta variable si bien es cierto no depende directamente del HSC, si genera un aumento en el tiempo de espera para ellos, ya que no se redireccionan las interconsultas con la frecuencia adecuada, lo que constituye un nudo crítico.

Para las tres formas de ingreso mencionadas anteriormente, el personal del Departamento de Gestión del usuario (DGU) es el encargado de jerarquizar las interconsultas, mediante un fundamento clínico que realiza el médico tratante en APS, CAE y AP, generando de esta manera una citación más expedita que suele demorar dos días. De no tener prioridad, se notifica al departamento de estadísticas sobre aquella interconsulta, la cual es ingresada al Repositorio Nacional de Listas de Espera (RNLE), otorgando una hora de atención por antigüedad de la solicitud, esta última opción demora entre 3 a 5 días. El DGU es quien realiza la citación a través de un llamado telefónico, contactando al centro de atención primaria o por medio de Radios locales. Esta actividad se realiza dos veces, en caso de fracasar el contacto de primera instancia, lo que constituye un nudo crítico. A continuación, se presenta mediante un diagrama de flujo el proceso de atención en la especialidad ginecológica:

\section{Diagrama de Flujo 1. Atención de especialidad ginecológica}

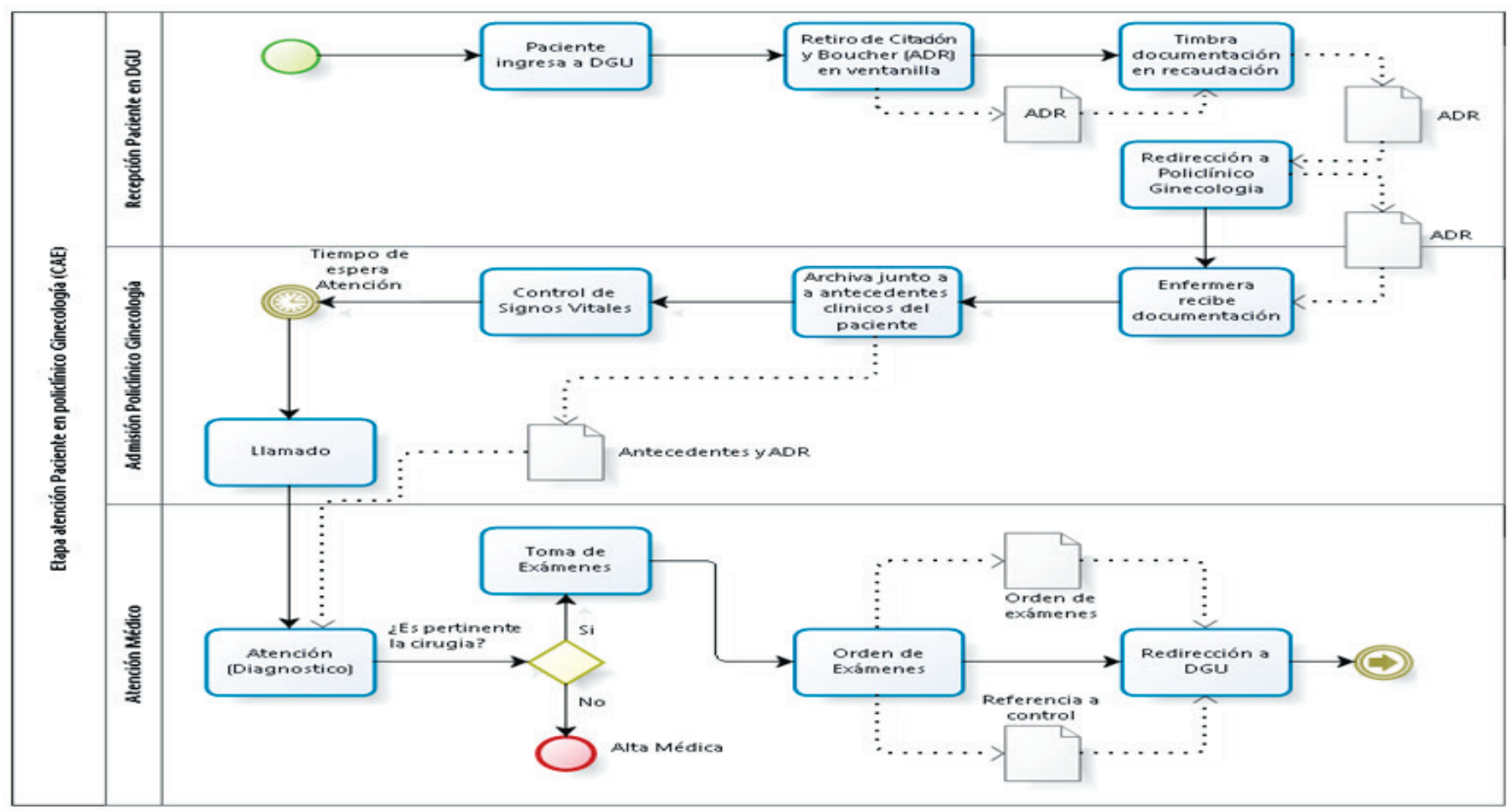

Fuente: Elaboración propia

Una vez analizados y cuantificados los tiempos de espera en cada etapa del proceso, se detectó que aquel más extenso es para la toma de ecografías, siendo este un nudo crítico muy significativo, tal como se muestra en el flujo $\mathrm{N}^{\circ} 2$ (puesto que esta es una lista de espera distinta a la primera atención con especialista), existiendo incluso ecografías pendientes desde el año 2009 a la fecha de la investigación año 2015, según la información proporcionada por el departamento de estadísticas HSC. La principal causa de este nudo crítico es la capacidad ociosa del ecógrafo, puesto que se toman exámenes solo en una jornada. Existen cinco especialistas 
ginecológicos que trabajan en el HSC y solo dos de ellos son los encargados de tomar ecografías mediante órdenes. Otro de los factores asociados a la toma de este examen, es la calidad del ecógrafo, si bien es cierto existe una sala de ecografías, el equipo con el cual cuenta el HSC es de muy baja calidad. A continuación, se presenta en el diagrama de flujo 2 el orden del proceso de toma de exámenes:

\section{Diagrama de Flujo 2. Orden y toma de exámenes}

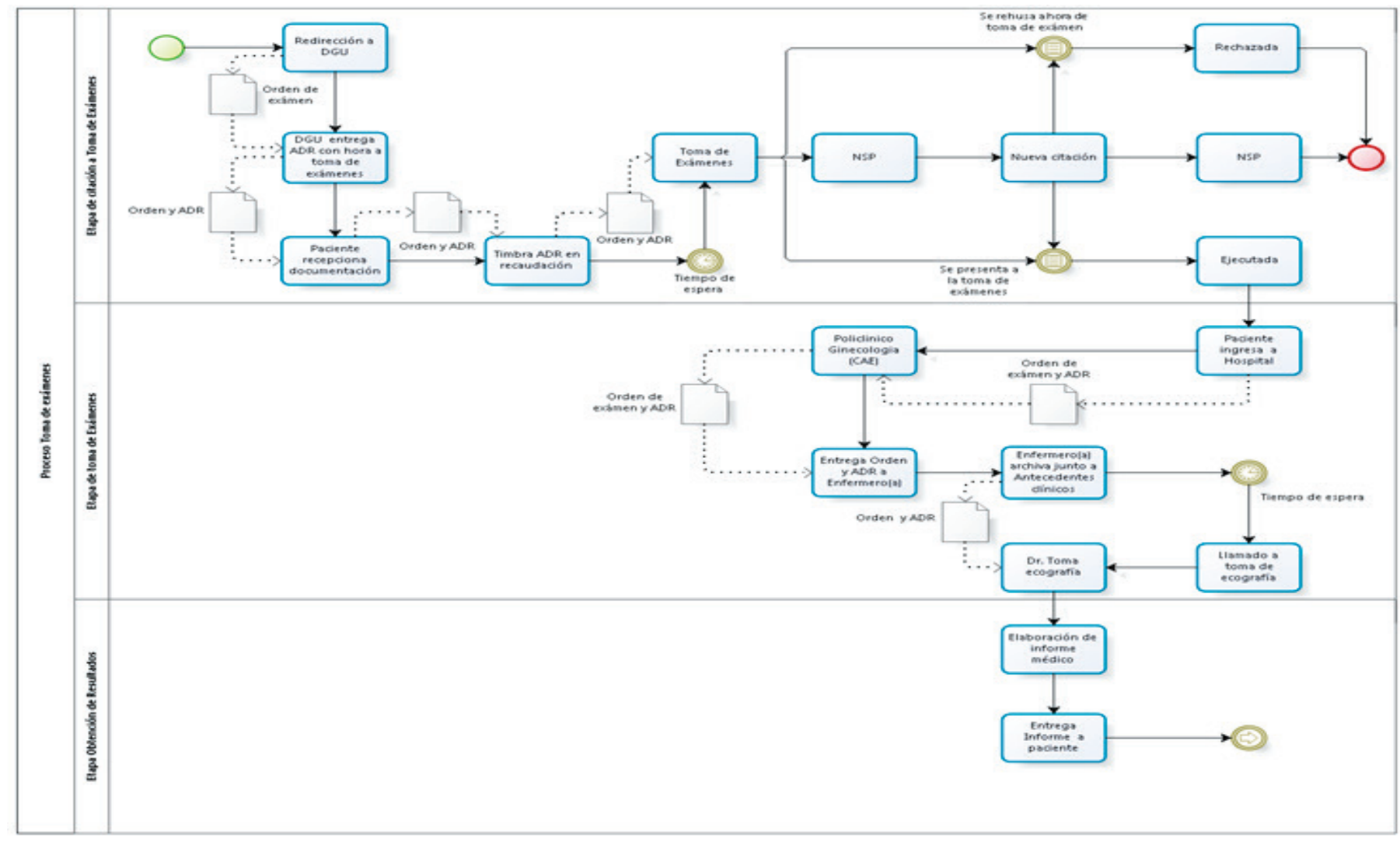

Fuente: Elaboración propia

Una vez que la paciente se ha realizado los exámenes (sangre, orina y ecografía), se le da una nueva hora de atención con el médico especialista (de preferencia con aquel que le solicitó los exámenes). Cabe mencionar que este tiempo de espera, en esta nueva lista es considerado razonable, de 2 a 5 días. Con la revisión de los exámenes, puede resultar que la paciente sea dada de alta, quede con algún tratamiento, o bien sea derivada a cirugía, en este último caso nuevamente ingresará a una lista de espera para intervención.

Además, se observó que la distribución del pabellón para cirugía depende de dos factores: los insumos disponibles y la disponibilidad de anestesistas (flujo $\mathrm{N}^{\circ} 3$ ), lo que se constituye en otro nudo crítico, que para este caso está provocando la subutilización de pabellones o el aplazamiento de una intervención, lo que finalmente se traduce en un tiempo de espera mayor para aquel paciente que está en esta lista de espera. A continuación, se presenta la distribución de pabellón en el diagrama de flujo 3: 


\section{Diagrama de flujo 3. Distribución de pabellón.}

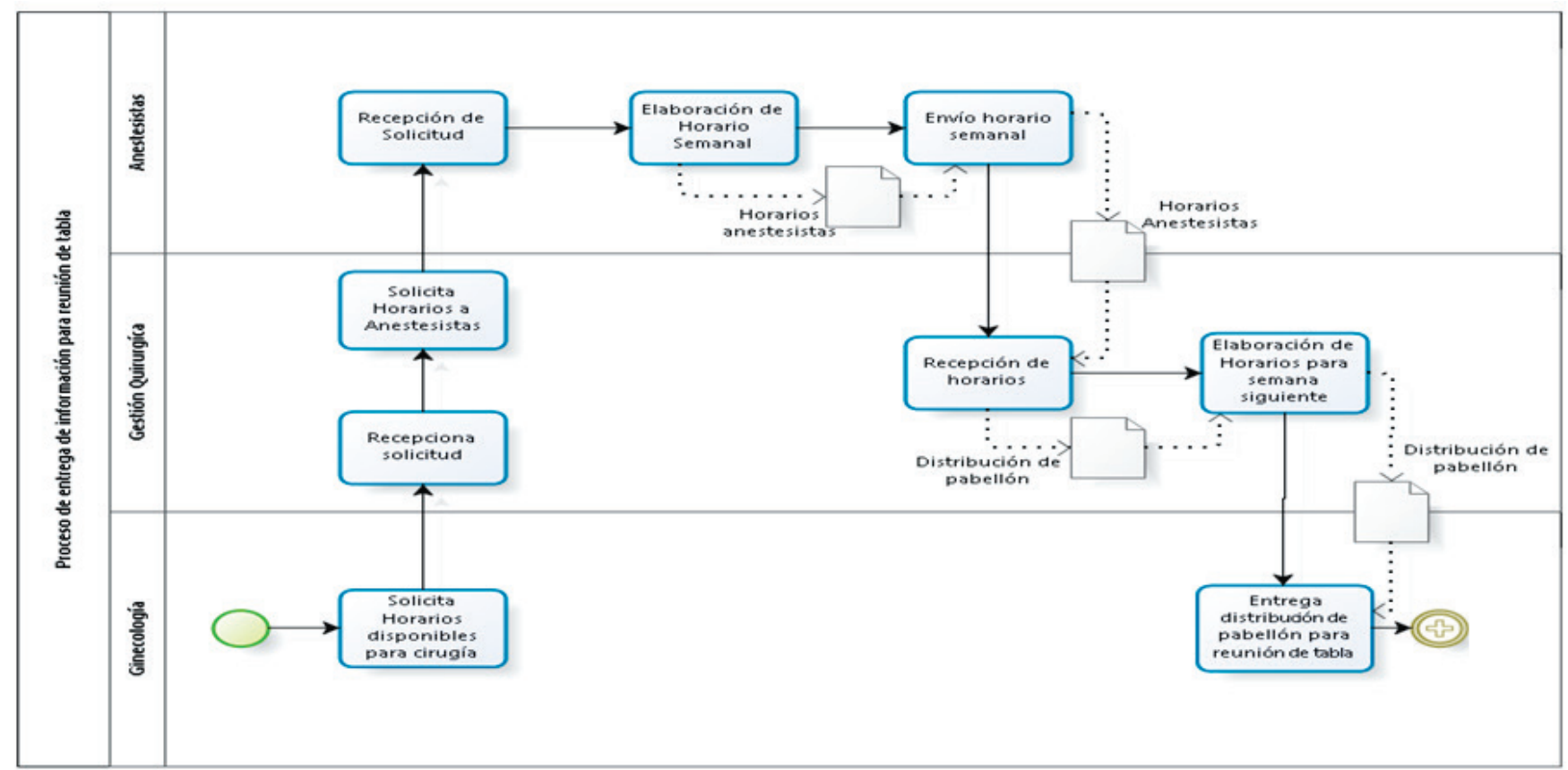

Fuente: Elaboración propia

\subsection{Propuesta}

En el nudo crítico de aquellos consultorios cuyo sistema no es compatible con el del hospital, es necesario que con urgencia el HSC pueda integrar a un sistema único esta nueva red geográfica de centros de salud.

Para la solución del nudo crítico correspondiente a la ecografía, se proponen dos vías de solución, una temporal y otra permanente. En el primer caso, la solución temporal consiste en la contratación de horas adicionales de dos médicos ginecólogos para toma de ecografías, en los horarios donde el ecógrafo no es utilizado, de las usuarias que están en lista de espera y que aún no se les ha tomado este examen. Además del o los ginecólogos, se debe contratar a una secretaria administrativa como asistente, para así agilizar el proceso. El principal beneficio de contratar horas para ecografías es que la lista de espera de ecografías se verá disminuida. Una segunda solución, para las atenciones futuras, se propone que, en la primera consulta con especialista, el médico pueda realizar una atención integrada, esta atención deberá constar de un examen físico y la toma de inmediato de la mencionada ecografía. La solución de este nudo crítico reduce muy significativamente la demora de atención, pero además permite un mejor diagnóstico, a tiempo, lo que a su vez incide en una disminución de la cantidad de mujeres que requerirían de cirugía, ya que su tratamiento sería más oportuno.

Respecto al ecógrafo, es importante la implementación de uno nuevo con el fin de entregar una mejor atención a los pacientes y también una buena herramienta de trabajo para los especialistas ginecológicos, logrando diagnósticos con mayor precisión.

En el caso del nudo crítico de falta de anestesistas, se propone con urgencia la contratación de más horas anestesista, en primer lugar, la compra de servicios, como medida de corto plazo, mientras se logra aumentar la planta con más especialistas en esta área de la medicina, cómo medida de solución permanente.

\section{Conclusiones}

Una vez revisados los procedimientos, se identificaron los nudos críticos que producen el aumento del tiempo de espera, es importante recalcar que una propuesta de mejora adecuada debe ser acorde a la realidad y que sean los propios funcionarios quienes participen en la solución a los problemas detectados, tal como se hizo en el HSC.

La atención integrada de salud (examen físico y toma de exámenes) en la primera citación es relevante en la actualidad, con ello se logra una reducción de tiempo importante ya que se eliminarán actividades como nuevas citaciones, nuevos registros, etc. Además, se reducen los costos puesto que se ahorran horas de trabajo y recursos materiales. Este cambio en el proceso permite disminuir considerablemente el tiempo de espera de los pacientes y, por ende, se logra una respuesta más oportuna, lo cual redunda en una mayor satisfacción de los 
mismos. Con lo anterior. se contribuye a lo que se espera del sistema de salud público: una mejor relación costo-efectividad.

Un aspecto adicional para disminuir el tiempo de espera en el POP es la incorporación de un nuevo ecógrafo y el aumento de su uso, ya que, en la primera consulta con especialista, el médico realizaría una atención integrada, esta atención deberá constar de un examen físico y la toma de examen, lo cual mejora ostensiblemente el pronto diagnóstico y hace más certero y a tiempo el respectivo tratamiento, lo que implica, en definitiva, en un menor tiempo de espera.

Otro nudo crítico lo constituye la falta de anestesistas, fundamentales en aquellos pacientes que requieren de cirugía, por lo que se necesita con urgencia la contratación de más de estos especialistas, aumentando así las horas de uso de los pabellones y por ende reduciendo los tiempos de espera.

Finalmente, se puede afirmar que toda organización debe ser capaz de identificar sus procesos y tareas, con el fin de conocer de manera detallada y descriptiva cada una de las actividades que se realizan. Partiendo de esta base, es más fácil identificar todo aquel proceso que no se ejecute de manera eficiente, siendo más fácil conocer los puntos críticos que permitan una propuesta de mejora que sea capaz de responder a todos los requerimientos y contribuir al logro de sus objetivos. 


\section{Referencias}

1. Letelier A. y Cifuentes, G. Situación y abordaje de lista de espera en un establecimiento de salud público en Santiago de Chile. Medwave. [Internet ]2014; 14(6). T[Consultado 2020 Jun 12 ]Disponible en: http://www.medwave.cl/link.cgi/ Medwave/Enfoques/ProbSP/6000?ver=sindiseno

2. Bernal, E. y De Las Torres, C. ¿Ha llegado la hora de la gestión de las listas de espera?. Gac Sanit. 2002; 16(5): p.4363.

3. Martí, J. Escobar Et al.La gestión de las listas de espera quirúrgicas por los centros sanitarios y los profesionales. Gac Sanit. 2002; 16(5):p. 440-3.

4. Martí-Valls, J.; Ballesta, E; González, R.; Solé, M. y Torras, G.. Resultados de un plan de gestión de listas de espera quirúrgica de prótesis articulares. Gac. Sanit. 2006;20(3):p. 248-2.

5. Rodríguez, J., Dackiewicz, N., \& Toer, D. La gestión hospitalaria centrada en el paciente. Arch Argent Pediatr. 2014;112(1):p. 55-3

6. Seguel, F., \& Paravic, T. Unidad de Investigación en Hospitales Autogestionados. Rev. Cien. y Enf. 2011; 17(2):p. 19-6.

7. Marti J. La gestión de las listas de espera quirúrgicas por los centros sanitarios y los profesionales. Gac. Sanit. 2002;16(5):p. 440-3.

8. Gutiérrez, A.; Solórzano, M.; Trujillo, C. y Plata, M. Prevalencia de prolapso del piso pélvico en pacientes con incontinencia urinaria de esfuerzo. Rev. Urol. Colom. 2012. XXI(3):p. 41-8.

9. Sung V, Hampton B. Epidemiology of pelvic floor dysfunction. Obstet Gynecol Clin North Am. 2009; 36(3):p. 421-2.

10. Flores, C., \& Pizarro, J. Calidad de vida en mujeres con alteraciones del piso pélvico: revisión de la literatura. Rev. Chil. Obstet. Ginecol. 2012; 77(3):p.175-7.

11. Ministerio de Salud. Repositorio Nacional de Listas de Espera (RNLE). 31.05.2015

12. Hospital de San Carlos. Bases de datos: Sinetsur; Producción quirúrgica de Pabellón Quirúrgico; Exámenes. 31.05.15

13. Osorio, J., y Paredes, E. (2001). Reingeniería de procesos en los Hospitales Públicos: ¿Reinventando la rueda? Rev. Esp. Salud Públ. 2001; 75:p.193-13.

14. Bernardes, A., Cecilio, L., Martinez, Y., Silvia, C., \& Bernardes de Carvalho, M. Modelo de gestión colegiada y descentralizada en hospital público: la óptica del equipo de enfermería. Rev. Latino-Am. Enfermagem. 2011; 19(4):p. 1-7.

15. Del Castillo Rueda, A., \& De Portugal Álvarez, J. Proyecto técnico de gestión y funcionamiento de la unidad asistencial de Medicina Interna. Anales de Med. Int. 2004; 21(1):p. 31-7

16.Sandoval, G. Internistas, cirujanos y anestesistas son los médicos con mayor déficit en red pública. Diario La Tercera. [Consultado 2014 Nov 14 ]Disponible en:http://www.latercera. com/noticia/nacional/2014/11/680-604502-9-internistascirujanos-y-anestesistas-son-los-medicos-con-mayor-deficiten-red.shtml

17.-Mañalich, J. ¿Por qué faltan médicos en Chile?: El mostrador. [Consultado 2014 Sep 25] Disponible en: http:// www.elmostrador.cl/noticias/opinion/2014/09/25/por-quefaltan-medicos-en-chile/.

18.- Khosravi, P. y Del Castillo, A. Reingeniería en el proceso de gestión e innovación de la asistencia médica hospitalaria. Anal. de Med. Int. 2005; 22(11):p. 509-1.

19.- Martí J. Las largas esperas en la atención sanitaria pública, un problema de pérdida de calidad. Rev. Electr. de geog. y cienc. Soc. 2008. XII(270) (102).

20.-Braun, H., Rojas, I., González, F., Fernández, M., y Ortiz, A. Prolapso genital severo: consideraciones clínicas, fisiopatológicas y de técnica quirúrgica al momento de su corrección. Rev. Chil. de Obst. y ginec. 2004; 69(2):p.149-7. 